\title{
(2) OPEN ACCESS \\ Intravitreal conbercept for diabetic macular oedema: 2-year results from a randomised controlled trial and open-label extension study
}

\author{
Kun Liu, ${ }^{1,2}$ Hanying Wang, ${ }^{1,2}$ Wei He, ${ }^{3}$ Jian Ye, ${ }^{4}$ Yanping Song ${ }_{1}^{5}$ Yusheng Wang ${ }_{1}^{6,7}$ \\ Xiaoling Liu, ${ }^{8}$ Zhifeng Wu, ${ }^{9}$ Shaojun Chen, ${ }^{10}$ Ke Fan, ${ }^{11}$ Yuling Liu, ${ }^{12}$ Feng Zhang, ${ }^{13,14}$ \\ Zhiqing Li, ${ }_{1}^{15}$ Lin Liu, ${ }_{16}^{16}$ Junjun Zhang, ${ }_{1}^{17}$ Xuedong Zhang, ${ }_{1}^{18}$ Junjie Ye, ${ }^{19}$ Xiaoling Liang, ${ }^{20}$ \\ Xiaoxin $\mathrm{Li}_{1}{ }^{21}$ Xiao Ke, ${ }_{1}^{22}$ Quan $\mathrm{Wu}_{1}{ }^{22} \mathrm{Jie} \mathrm{Li}_{1}{ }^{23}$ Shanshan Tao, ${ }^{23}$ Xinguo Wang, ${ }^{22}$ \\ Philip Rosenfeld, ${ }^{24}$ Jeffrey $S$ Heier, ${ }^{25}$ Peter Kaiser, ${ }^{26}$ Xun Xu ${ }^{1,2}$
}

- Additional supplemental material is published online only. To view, please visit the journal online (http://dx.doi. org/10.1136/bjophthalmol2020-318690).

For numbered affiliations see end of article.

\section{Correspondence to} Dr Xun Xu, Shanghai General Hospital Department of Ophthalmology (aka National Clinical Research Center for Eye Diseases), Shanghai, China; drxuxun@sjtu.edu.cn

$\mathrm{KL}$ and $\mathrm{HW}$ contributed equally.

Received 7 January 2021 Revised 31 March 2021 Accepted 13 April 2021

\section{Check for updates}

(c) Author(s) (or their employer(s)) 2021. Re-use permitted under CC BY-NC. No commercial re-use. See rights and permissions. Published by BMJ.

To cite: Liu K, Wang $\mathrm{H}$, He W, et al. Br I Ophthalmol Epub ahead of print: [please include Day Month Year]. doi:10.1136/

bjophthalmol-2020-318690

\begin{abstract}
Background To demonstrate the efficacy and safety of intravitreal injections of conbercept versus laser photocoagulation in the treatment of diabetic macular oedema (DME).

Methods A 12-month multicentre, randomised, double-masked, double-sham, parallel controlled, phase III trial (Sailing Study), followed by a 12-month open-label extension study. Patients with centreinvolved DME were randomly assigned to receive either laser photocoagulation followed by pro re nata (PRN) sham intravitreal injections (laser/sham) or sham laser photocoagulation followed by PRN $0.5 \mathrm{mg}$ conbercept intravitreal injections (sham/conbercept). Patients who entered the extension study received PRN conbercept treatment. The primary endpoint was the changes in best-corrected visual acuity (BCVA) from baseline.
\end{abstract}

Results A total of 248 eyes were included in the full analysis set and 157 eyes continued in the extension study. Significant improvement in mean change in BCVA from baseline to month 12 was observed in the sham/conbercept group (8.2 \pm 9.5 letters), whereas no improvement was observed in the laser/sham group $(0.3 \pm 12.0$ letters). Patients in the laser/sham group showed a marked improvement in BCVA after the switch to conbercept in the extension study, and there was no difference in BCVA between the two groups at the end of the extension study.

Conclusion The use of a conbercept PRN intravitreal injection regimen improved the BCVA of patients with $\mathrm{DME}$, and its efficacy was better than that of laser photocoagulations, and the same efficacy was observed when the eyes treated with laser alone were switched to conbercept.

Trial registration number NCT02194634.

\section{INTRODUCTION}

Diabetic retinopathy and diabetic macular oedema (DME) are the most common causes of preventable blindness worldwide, ${ }^{1-3}$ and the worldwide prevalence of DME is $4.7 \%-10.1 \%{ }^{4-6}$ Retinal thickening and hard exudates that threaten or involve the centre of the macula are considered to have clinical significance. $^{78}$
For decades, focal and grid laser photocoagulation has been the mainstay treatment to prevent vision loss. However, the intraocular administration of antivascular endothelial growth factor (VEGF) agents reduces DME without tissue damage and is more effective in improving vision. ${ }^{1-3}$ Due to its advantages, currently, anti-VEGF agents has become the gold standard for the treatment of DME.

VEGF has been shown to have a major role in promoting exudation and neovascularisation, ${ }^{9-11}$ and the inhibition of VEGF can stabilise or even reverse the retinal damage due to DME. ${ }^{12}$ Over the past two decades, anti-VEGF agents such as pegaptanib, bevacizumab, ranibizumab and aflibercept were approved for the treatment of ocular diseases that involve retinal neovascularisation and exudation, including DME. ${ }^{13-17}$

Conbercept (KH902; Chengdu Kanghong Biotech Co., China) is a recombinant fusion protein with key domains 2, 3 and 4 from VEGF receptors 1 and 2. It has a high affinity for all VEGF isoforms and for placental growth factor. ${ }^{18}$ In 2013, it was approved in China for the treatment of neovascular (wet) age-related macular degeneration. ${ }^{18}$ In addition, conbercept is approved for the treatment of choroidal neovascularisation secondary to pathological myopia. The objective of the Sailing Study was to compare the efficacy and safety of intravitreal conbercept injections versus laser for the treatment of DME. This report includes the 1-year results of the Sailing Study and the 1-year results of its extension study in which patients crossed over to conbercept therapy.

\section{METHODS}

\section{Study design and patients}

The Sailing Study was a multicentre, randomised, double-masked, double-sham, parallel controlled, phase III trial (registered at ClinicalTrials.gov) conducted at 18 centres in China. An open-label extension study was conducted after patients completed the Sailing Study.

For each patient, only one eye was enrolled in the study. If both eyes of a subject met the inclusion criteria, the investigators determined the target eye from the medical perspective, with the eye with poorer vision selected in principle. The 
key inclusion criteria were (1) >18 years of age; (2) type I or II diabetes mellitus; (3) haemoglobin A1c (HbA1c) of $<10 \%$; and (4) the study eye had to meet the following criteria: (i) DME involving the central fovea, (ii) ETDRS best-corrected visual acuity (BCVA) between 73 and 24 letters (Snellen equivalent of 20/40-20/320), (iii) central retinal thickness (CRT) of $>300 \mu \mathrm{m}$ according to optical coherence tomography (OCT) imaging, (iv) clear ocular media and adequate pupil dilation for examination and imaging and (5) the ETDRS BCVA of the subject's non-target eye of $\geq 24$ letters (equivalent to 20/320 of the Snellen vision). The key exclusion criteria were (1) active eye infection in either eye; (2) any ophthalmic conditions leading to macular oedema or alterations in vision other than diabetic retinopathy; (3) panretinal photocoagulation within 6 months prior to screening or local/grid retinal photocoagulation within 3 months prior to screening; (4) treatment with anti-VEGF drugs (eg, aflibercept, pegaptanib sodium, ranibizumab, bevacizumab, etc) within 6 months prior to screening; (5) any type of intraocular surgery (eg, cataract surgery, yttrium aluminium garnet (YAG) posterior capsulotomy, etc) within 3 months prior to screening; (6) uncontrolled hypertension; and (7) stroke, transient ischaemic attack, myocardial infarction or acute congestive heart failure within 6 months prior to screening. The detailed exclusion criteria are shown in the online supplemental appendix.

\section{Randomisation and masking}

During the Sailing Study, the eligible patients were randomly assigned in a 1:1 ratio to receive either sham laser followed by conbercept (sham/conbercept group) or laser followed by a sham injection (laser/sham group) according to the interactive web response system. Masking was performed for the patients, masked investigators and statisticians. Treatments were performed by unmasked investigators who were not involved in any other study work. The extension study was an open-label study, so no masking was necessary.

\section{Treatments}

During the Sailing Study, the patients in the sham/conbercept group received sham laser and then an intravitreal injection of conbercept (0.5 mg, Chengdu Kanghong Biotech Co.) on day 0, followed by pro re nata (PRN) conbercept treatments and sham laser treatments during the monthly follow-up per predefined criteria. Patients in the laser/sham group received modified ETDRS grid photocoagulation and then a sham intravitreal injection on day 0 . Starting at month 3 , the laser group received PRN sham injections and active laser treatments during the monthly follow-up per predefined criteria, but the retreatment of laser will not be evaluated if less than 12 weeks from the last sham or active laser treatment. When the patients received active PRN conbercept or laser, the sham laser or sham injection was performed on the same day. An interval of at least 2 hours was ensured between the active or sham laser treatment and the active or sham intravitreal injection.

PRN laser was performed if any of the following criteria was met: (1) retinal thickening within a radius of $500 \mu \mathrm{m}$ around the centre of the macula; (2) hard exudates with adjacent retinal thickening within a radius of $500 \mu \mathrm{m}$ around the centre of the macula (for hard exudates left behind after retinal thickening regression, no treatment is required); (3) the total area of one or multiple regions with retinal thickening is no less than that of one optic disk $\left(2.54 \mathrm{~mm}^{2}\right)$; and the distance from a part of the region of retinal thickening to the centre of the macula is below a diameter of the optic disk $(1800 \mu \mathrm{m})$.
The criteria for repeated conbercept treatments were (any of the following criteria satisfied): (1) CRT increased by at least 50 $\mu \mathrm{m}$ if compared with the previous minimum value; (2) CRT of $\geq 300 \mu \mathrm{m}$; (3) cystoid degeneration of retina, subretinal fluid or pigment epithelial detachment in the macular region; (4) ETDRS BCVA was improved by at least five letters if compared with that during the previous visit; and (5) ETDRS BCVA declined by at least five letters if compared with the maximum number of letters previously, combining with CRT increase, in comparison with that when ETDRS BCVA is at its optimum level.

Other treatments forbidden from the study included (1) antiVEGF drugs other than conbercept; (2) any other antiangiogenic drugs; (3) ocular or systemic steroids; (4) drugs with toxicity to the lens, retina or nerves; (5) anticoagulant or antiplatelet therapy, except for low-dose prophylactic use; or (6) treatments to the study eye with impact on the efficacy and safety assessments of the present study, such as panretinal photocoagulation, verteporfin photodynamic therapy, external beam radiotherapy, vitrectomy, transpupillary thermotherapy and macular surgery.

Starting at month 6 , whether the patients needed rescue treatment was assessed monthly. If patients met either one of the criteria for rescue treatment: (1) ETDRS BCVA declined by at least 15 letters compared with the maximum number of letters previously and lowered to a degree below the baseline; or (2) ETDRS BCVA decreased to a level that at least 10 letters fewer than the baseline during visits of two consecutive months, and the investigators believed that the visual impairment was caused by the persistent or worsening oedema from the DME, then their sham designation was switched to active treatment in whatever group they were assigned.

During the extension study, patients in both groups received PRN conbercept treatment with monthly follow-up.

\section{Assessment}

The primary endpoint of the Sailing Study was the mean change in BCVA from baseline to month 12. Secondary endpoints included the change in CRT from baseline to month 12 and safety. Safety assessments included both ocular and non-ocular adverse events (AEs) and serious adverse events (SAEs). Other endpoints included changes in CRT, total macular volume (TMV), fluorescein angiographic leakage area, BCVA and the total score of 25-Item National Eye Institute Visual Function Questionnaire (NEI VFQ-25) from baseline to month 12, as well as the proportion of $\leq 5,10$ and 15 letters vision gain or loss from baseline to months 6 and 12 .

The primary outcome of the extension study was the mean change in BCVA from month 12 to 24. Secondary outcomes included the long-term safety of conbercept, change in BCVA from month 12 to 24 , change in CRT from month 12 to 24, change in TMV from month 12 to 24 , change in leakage area from month 12 to 24 and the number of injections in the extension study.

Patients were followed up monthly for ophthalmological examinations, including ETDRS protocol BCVA, intraocular pressure, slit-lamp examination and OCT. Colour fundus photography (CFP) and fundus fluorescein angiography (FFA) were performed every 3 months. NEI VFQ-25 was completed every 6 months. Protocol BCVA was measured by using the standard ETDRS visual acuity chart (starting at the $4 \mathrm{~m}$ testing distance). All study-related image aqcuisitions were certificated by EyeKor, LLC (online supplemental table 1). OCT, CFP and FFA images were assessed by the reading centre of the University of Wisconsin-Madison. 


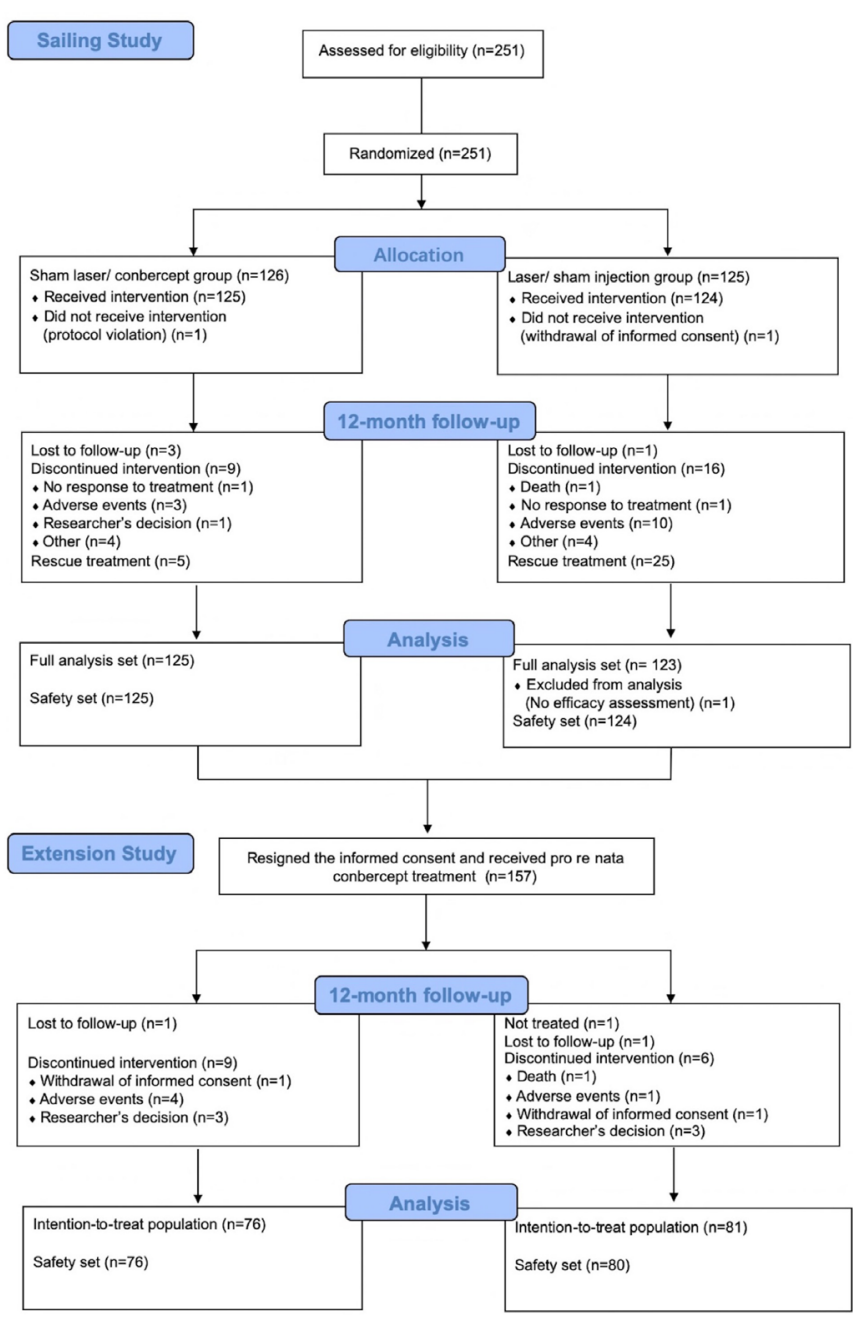

Figure 1 Study flowchart.

\section{Statistical analysis}

Assuming that the changes in BCVA from baseline to month 12 had a difference of five letters between the two groups, the SD of the two groups was 10 and 12 letters, respectively. A total of 208 patients (104 in each group) were calculated with a one-sided significance level of $2.5 \%$ and a power of $90 \%$. Assuming the drop-out rate of 20\%, 124 patients were needed in each group.

The full analysis set in the Sailing Study included all randomised patients who received at least one treatment with the corresponding efficacy assessment. The safety set (SS) in the Sailing Study included all patients who received at least one treatment and at least one safety assessment. The intention-totreat population in the extension study included all patients who agreed to continue the follow-up and received at least one extension assessment. The SS in the extension study included all patients who received at least one conbercept treatment during the 2-year period.For the primary endpoint of the Sailing Study, the missing values were imputed using the last observation carried forward (LOCF) method. For patients who had a rescue treatment, the missing values were imputed using LOCF method prior to the first rescue treatment. Missing values for the secondary endpoints in the Sailing Study and all outcomes in the extension study were not imputed.

Statistical analyses were performed using SAS V.9.4. Continuous variables were presented as means \pm SD. The analysis of covariance was used for the primary outcome comparison. For secondary efficacy outcomes, the independent t-test was used for intergroup comparisons and a paired t-test was used for comparisons between two time points. Categorical variables were presented as frequencies (percentages) and compared with the Cochran-Mantel-Haenszel $\chi^{2}$ test or Fisher's exact test, as appropriate. A two-sided $\mathrm{p}$ value of $<0.05$ was considered statistically significant.

\section{RESULTS \\ Patient disposition, baseline characteristics and treatment experience}

Between August 2014 and December 2015, a total of 251 eligible patients were randomised. After completing the Sailing Study, 157 patients enrolled in the extension study. Figure 1 shows the study flowchart and analysis sets.

The baseline characteristics of the patients in the Sailing Study were well balanced between groups (table 1). The patient disposition in the extension study is shown in online supplemental table 2. Eyes in the Sailing Study received an average of 2.6 and 2.7 sham or laser treatments in the sham/conbercept group and laser/sham groups, respectively, and 9.5 and 9.7 intravitreal or sham injections, respectively. More patients in the laser group required a rescue treatment compared with the conbercept group in the Sailing Study $(20.3 \%$ vs $4.0 \%, \mathrm{p}<0.001)$. During the extension study, the numbers of conbercept treatments were $8.5 \pm 3.5$ and $8.6 \pm 3.4$ in the conbercept and laser groups, respectively (online supplemental table 3 ).

\section{Efficacy}

Significant improvement in BCVA from baseline to month 12 was observed in the conbercept group $(8.2 \pm 9.5$ letters, $\mathrm{p}<0.001$ ), whereas no improvement was observed in the laser group $(0.3 \pm 12.0$ letters, $\mathrm{p}=0.810)$ (figure $2 \mathrm{~A})$. The changes in BCVA from baseline were significantly different between the two groups at all time points during the first year (all $\mathrm{p}<0.001$ ). The subset of eyes that continued in the extension study also showed a similar result during the first year (figure 2B). During the extension study, after patients in the laser group crossed over to receiving PRN conbercept treatment, there was a significant improvement in BCVA at all time points of the second year (vs month 12, all $\mathrm{p}<0.05$ ) (figure $2 \mathrm{~B}$ ). The change in BCVA at month 24 in the laser group was $4.9 \pm 9.4$ letters from month $12(\mathrm{p}<0.001)$ and $8.0 \pm 11.4$ letters from baseline $(\mathrm{p}<0.001)$. For patients in the conbercept group, 2-year results showed that they continued to maintain visual acuity gains from intravitreal injection. The changes in BCVA at month 24 in the sham conbercept group were $2.3 \pm 8.8$ letters from month $12(p=0.030)$ and $8.3 \pm 12.4$ letters from baseline $(\mathrm{p}<0.001)$.

Both the results of 6 months and those of 12 months showed that significantly more eyes treated with conbercept gained vision from baseline, whereas significantly more eyes treated with laser lost letters from baseline (all $\mathrm{p}<0.001)$ (online supplemental table 4). The proportion of eyes with vision gain of $\geq 15$ letters from baseline were $17.4 \%(21 / 121)$ and $25.0 \%(28 / 112)$ at months 6 and 12 in the sham/conbercept group compared with $6.8 \%(8 / 118)$ and $14.9 \%(13 / 87)$ in the laser/sham group, respectively, at the same time points. The proportion of eyes with vision loss of $\geq 15$ letters from baseline were $1.7 \%(2 / 121)$ and $3.4 \%(4 / 112)$ at months 6 and 12 in the sham/conbercept group compared with $0 \%(0 / 118)$ and $1.1 \%(1 / 87)$ in the laser/ sham group, respectively.

Significant differences in CRT were observed beteween the two groups at all time points in the first 12 months (all $p<0.01$ ) 
Table 1 Baseline characteristics of patients in the Sailing Study

\begin{tabular}{|c|c|c|c|c|c|}
\hline \multirow[b]{2}{*}{ Variables } & \multicolumn{2}{|c|}{ Conbercept $(95 \% \mathrm{Cl})$} & \multicolumn{2}{|l|}{ Laser $(95 \% \mathrm{Cl})$} & \multirow[b]{2}{*}{$P$ value } \\
\hline & $(n=125)$ & & $(n=123)$ & & \\
\hline Age (years) & $58.9 \pm 8.5$ & (57.43 to 60.42 ) & $58.7 \pm 8.8$ & (57.18 to 60.30$)$ & 0.863 \\
\hline Sex, n (\%) & & & & & 0.527 \\
\hline Male & $66(52.8)$ & (51.31 to 53.27 ) & $60(48.8)$ & (47.27 to 49.23 ) & \\
\hline Female & $59(47.2)$ & (46.73 to 48.69$)$ & $63(51.2)$ & (50.77 to 52.73 ) & \\
\hline BMI $\left(\mathrm{kg} / \mathrm{m}^{2}\right)$ & $24.2 \pm 3.4$ & (24.13 to 24.50$)$ & $25.0 \pm 4.5$ & (24.83 to 25.32 ) & 0.095 \\
\hline Duration of diabetes (years) & $12.3 \pm 7.0$ & (12.16 to 12.92$)$ & $11.0 \pm 6.3$ & (10.77 to 11.44 ) & 0.138 \\
\hline $\mathrm{HbA1c}$ & $7.06 \pm 1.19$ & (6.85 to 7.27 ) & $7.13 \pm 1.192$ & (6.92 to 7.35 ) & 0.646 \\
\hline Study eye, n (\%) & & & & & 0.881 \\
\hline Right & $72(57.6)$ & (56.21 to 58.16 ) & $72(58.5)$ & (57.19 to 59.13 ) & \\
\hline Left & $53(42.4)$ & (41.84 to 43.79 ) & $51(41.5)$ & (40.87 to 42.81 ) & \\
\hline BCVA (ETDRS letter) & $56.6 \pm 11.5$ & (54.50 to 59.19 ) & $57.6 \pm 11.5$ & (54.59 to 59.62 ) & 0.505 \\
\hline IOP (mm Hg) & $15.2 \pm 3.0$ & (14.62 to 15.68 & $15.1 \pm 3.4$ & (14.45 to 15.66$)$ & 0.819 \\
\hline CRT $(\mu \mathrm{m})$ & $480.0 \pm 180.0$ & (476.36 to 495.90$)$ & $470.0 \pm 160.0$ & (464.09 to 481.28 ) & 0.753 \\
\hline $\operatorname{TMV}\left(\mathrm{mm}^{3}\right)$ & $9.9 \pm 1.2$ & (9.45 to 10.39 ) & $10.1 \pm 1.4$ & (9.68 to 10.72 ) & 0.534 \\
\hline Leakage area $\left(\mathrm{mm}^{2}\right)$ & $28.2 \pm 11.0$ & (26.20 to 30.11$)$ & $27.8 \pm 10.8$ & (25.92 to 29.76 ) & 0.820 \\
\hline NEI VFQ-25 total score & $65.9 \pm 17.1$ & (62.88 to 68.93$)$ & $66.7 \pm 17.9$ & (63.47 to 69.85$)$ & 0.733 \\
\hline History of laser treatments, $\mathrm{n}(\%)$ & & & & & 0.622 \\
\hline Yes & $77(61.60)$ & (60.14 to 62.06$)$ & $72(58.54)$ & (57.25 to 59.19$)$ & \\
\hline No & $48(38.40)$ & (37.94 to 39.86 ) & $51(41.46)$ & (40.81 to 42.75$)$ & \\
\hline History of ocular anti-VEGF treatments, $\mathrm{n}(\%)$ & & & & & 0.098 \\
\hline Yes & $14(11.20)$ & (10.08 to 11.30$)$ & $23(18.70)$ & (17.61 to 19.13$)$ & \\
\hline No & $111(88.80)$ & (88.70 to 89.92 ) & $100(81.30)$ & (80.87 to 82.39 ) & \\
\hline
\end{tabular}

BCVA, best-corrected visual acuity; BMI, Body Mass Index; CRT, central retinal thickness; ETDRS, Early Treatment Diabetic Retinopathy Study; HbA1c, haemoglobin A1c; IOP, intraocular pressure; NEI VFQ-25, 25-Item National Eye Institute Visual Function Questionnaire; TMV, total macular volume; VEGF, vascular endothelial growth factor.

(figure 3A). The mean reduction of CRT from baseline to month 12 was $200 \pm 210 \mu \mathrm{m}(\mathrm{p}<0.001)$ in the sham/conbercept group and $130 \pm 190 \mu \mathrm{m}(\mathrm{p}<0.001)$ in the laser/sham group. Significant reduction of TMV from baseline was observed by month 3

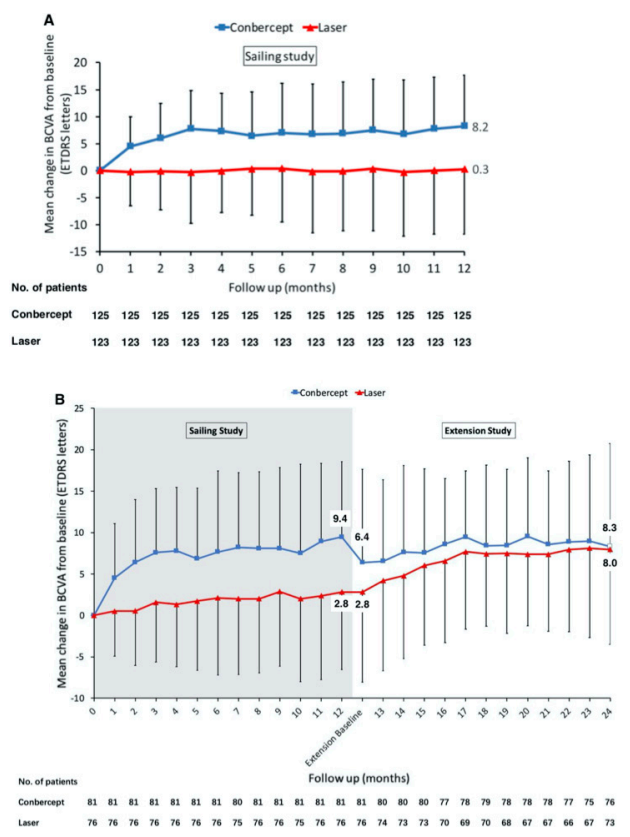

Figure 2 Mean changes in BCVA for laser and conbercept study groups in the Sailing Study. (A) One-year results compared with baseline. (B) Two-year results compared with baseline for the subset of eyes that continued in the extension study. Error bars denote SD. BCVA, best-corrected visual acuity. $\left(1.3 \pm 0.9 \mathrm{~mm}^{3}, \mathrm{p}<0.001\right)$ in eyes treated with sham/conbercept, whereas this reduction was observed at month $6\left(0.6 \pm 0.7 \mathrm{~mm}^{3}\right.$, $\mathrm{p}=0.004$ ) in eyes treated with laser/sham (figure $3 \mathrm{C}$ ). There was a significant difference in TMV between the two groups at months 3 and 6 (both $\mathrm{p}<0.05$ ), but not at months 9 and 12. The trends of the leakage area changes were similar to those of the CRT changes. The mean reduction of leakage area from baseline to month 12 was $7.9 \mathrm{~mm}^{2}(\mathrm{p}<0.001)$ in the sham/conbercept group and $3.9 \mathrm{~mm}^{2}(\mathrm{p}<0.001)$ in the laser/sham group, and the leakage area was significantly decreased in the sham/conbercept group compared with the laser/sham group $(\mathrm{p}<0.001)$. The mean change in the NEI VFQ-25 total score from baseline was significantly different between the sham/conbercept group and the laser/sham groups at months $6(4.4 \pm 16.0$ vs $-1.5 \pm 15.5$, $\mathrm{p}=0.004)$ and $12(4.3 \pm 19.5$ vs $-3.8 \pm 17.7, \mathrm{p}=0.001)$ (figure $3 \mathrm{E}$ ). During the extension study, the improvements in anatomical outcomes (CRT, TMV and leakage area) were maintained in both groups (figure 3B,D), and the laser group did better in reducing fluorescein leakage area.

\section{Safety}

In the Sailing Study, the proportion of total AEs $(87.2 \%$ vs $87.1 \%)$ and SAEs (17.6\% vs $19.4 \%$ ) were similar between the sham/conbercept and laser/sham groups (table 2). The most common AEs were visual impairment (20.0\%), upper respiratory tract infection $(20.0 \%)$, intraocular hypertension $(12.8 \%)$, hypertension $(12.0 \%)$, elevated blood pressure $(12.0 \%)$ and subconjunctival haemorrhage $(12.0 \%)$ in patients treated with conbercept. The most common AEs were visual impairment (19.4\%), upper respiratory tract infection (17.7\%), hypertension $(10.5 \%)$ and diabetic nephropathy $(10.5 \%)$ in patients treated with laser. More patients reported ocular AEs (57.6\%) in the 


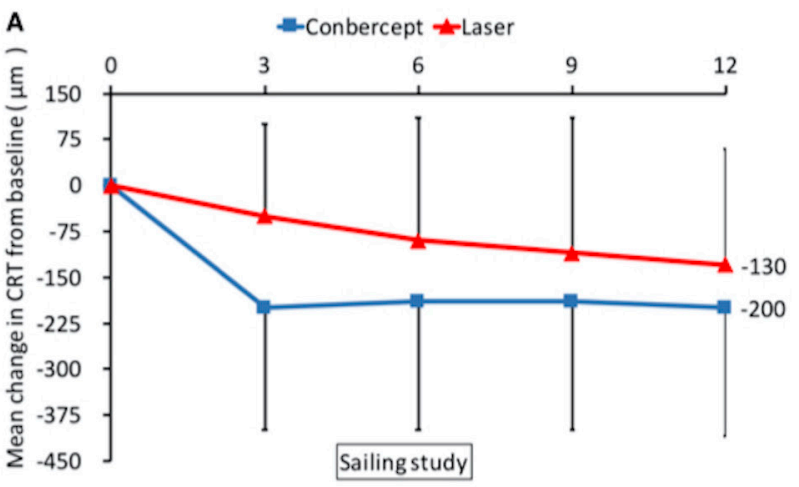

No. of patients

Conbercept 124

Laser $\quad 121$

C

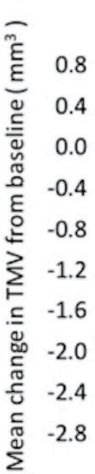

No. of patients

Conbercept 38

Laser
Follow up (months)

120

112
118

110
B

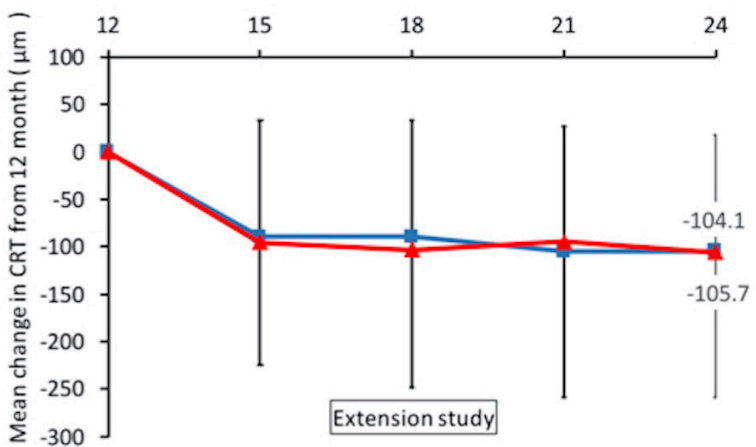

No. of patients

Follow up (months)

$\begin{array}{llllll}\text { Conbercept } & 75 & 70 & 68 & 66 & 72 \\ \text { Laser } & 80 & 71 & 75 & 76 & 75\end{array}$
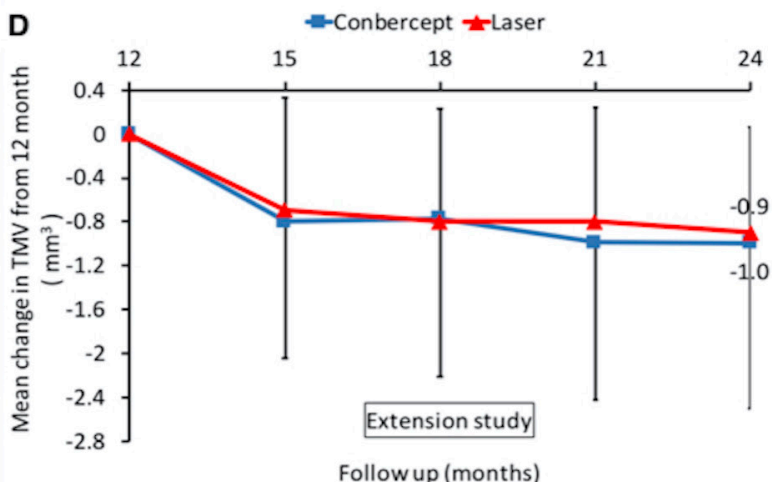

No. of patients

$\begin{array}{llllll}\text { Conbercept } & 75 & 70 & 68 & 66 & 72 \\ \text { Laser } & 80 & 71 & 75 & 76 & 75\end{array}$


Table 2 AEs in the Sailing Study and extension study

\begin{tabular}{|c|c|c|c|c|}
\hline & $\begin{array}{l}\text { Conbercept } \\
(n=125)\end{array}$ & $\begin{array}{l}\text { Laser } \\
(n=124)\end{array}$ & $\begin{array}{l}\text { Conbercept } \\
(n=76)\end{array}$ & $\begin{array}{l}\text { Laser } \\
(\mathrm{n}=80)\end{array}$ \\
\hline Events & \multicolumn{2}{|l|}{ Sailing Study } & \multicolumn{2}{|l|}{ Extension study } \\
\hline All AEs & $109(87.2)$ & $108(87.1)$ & $61(80.3)$ & $75(93.8)$ \\
\hline Treatment-related AEs & $45(36.0)$ & $19(15.3)$ & $30(39.5)$ & $34(42.5)$ \\
\hline SAEs & $22(17.6)$ & $24(19.4)$ & $11(14.5)$ & $16(20.0)$ \\
\hline Death & 0 & $1(0.8)$ & 0 & $1(1.3)$ \\
\hline All ocular AEs & $72(57.6)$ & 49 (39.5) & $39(51.3)$ & $50(62.5)$ \\
\hline $\begin{array}{l}\text { Treatment-related ocular } \\
\text { AEs }\end{array}$ & $40(32.0)$ & $12(9.7)$ & $27(35.5)$ & $31(38.8)$ \\
\hline Ocular SAEs & $6(4.8)$ & $2(1.6)$ & $7(9.2)$ & $4(5.0)$ \\
\hline \multicolumn{5}{|l|}{$\begin{array}{l}\text { AEs occurring in }>5 \% \text { of } \\
\text { patients in either group }\end{array}$} \\
\hline Visual impairment & $25(20.0)$ & $24(19.4)$ & $3(3.9)$ & $5(6.3)$ \\
\hline $\begin{array}{l}\text { Upper respiratory tract } \\
\text { infection }\end{array}$ & $25(20.0)$ & $22(17.7)$ & $9(11.8)$ & $15(18.8)$ \\
\hline Intraocular hypertension & $16(12.8)$ & $6(4.8)$ & $11(14.5)$ & $13(16.3)$ \\
\hline Systemic hypertension & $15(12.0)$ & $13(10.5)$ & $5(6.6)$ & $5(6.3)$ \\
\hline Elevated blood pressure* & $15(12.0)$ & $7(5.6)$ & $3(3.9)$ & $10(12.5)$ \\
\hline $\begin{array}{l}\text { Subconjunctival } \\
\text { haemorrhage }\end{array}$ & $15(12.0)$ & $4(3.2)$ & $2(2.6)$ & $4(5.0)$ \\
\hline Diabetic nephropathy & $9(7.2)$ & $13(10.5)$ & $1(1.3)$ & $5(6.3)$ \\
\hline Hyperlipaemia & $7(5.6)$ & $9(7.3)$ & $3(3.9)$ & $3(3.8)$ \\
\hline Urinary tract infection & $7(5.6)$ & $6(4.8)$ & $2(2.6)$ & $3(3.8)$ \\
\hline Elevated blood glucose & $6(4.8)$ & $7(5.6)$ & $6(7.9)$ & $2(2.5)$ \\
\hline Vitreous haemorrhage & $6(4.8)$ & $7(5.6)$ & $4(5.3)$ & $4(5.0)$ \\
\hline Nasopharyngitis & $6(4.8)$ & $6(4.8)$ & $4(5.3)$ & $2(2.5)$ \\
\hline Elevated blood urea & $5(4.0)$ & $7(5.6)$ & $1(1.3)$ & $2(2.5)$ \\
\hline Detected urine protein & $2(1.6)$ & $12(9.7)$ & $1(1.3)$ & $1(1.3)$ \\
\hline Xerophthalmia & $2(1.6)$ & $7(5.6)$ & $3(3.9)$ & $2(2.5)$ \\
\hline Vitreousopacity & 0 & $6(4.8)$ & $4(5.3)$ & $2(2.5)$ \\
\hline
\end{tabular}

*Elevated blood pressure but not enough to diagnose hypertension.

$A E$, adverse event; $S A E$, serious adverse event.

sham/conbercept group compared with $39.5 \%$ in the laser/sham group. Most of the additional ocular AEs with conbercept treatment were intraocular hypertension $(12.8 \%$ vs $4.8 \%$ with laser $)$ and subconjunctival haemorrhage $(12.0 \%$ vs $3.2 \%$ with laser). Endophthalmitis occurred in two patients treated with conbercept and one thereof was non-infectious intraocular inflammation. Endophthalmitis did not occur in patients treated with laser. The incidence of non-ocular SAEs in the laser/sham group (16.9\%) was higher than in the sham/conbercept (12.0\%). The incidences of Anti-Platelet Trialists' Collaboration-defined arterial thromboembolic events (APTC-ATEs) were equal (3.2\%) in both groups.

The AEs that occurred during the extension study were similar to those in the Sailing Study. Intraocular hypertension $(24 / 156$, $15.4 \%)$ and upper respiratory tract infection $(24 / 156,15.4 \%)$ were the most frequently reported AEs in the second year. For APTC-ATEs, rates were $7.7 \%(12 / 156)$ in the extention study. No death occurred in the conbercept group during the 2-year period. One patient in the laser group died from cerebral haemorrhage during the first year, and another patient died from ischaemic cardiomyopathy in the second year.

\section{DISCUSSION}

The Sailing Study was designed to evaluate the use of conbercept for the management of patients with centre-involved DME. The primary endpoint of the Sailing Study was the mean change in BCVA from baseline to month 12 . The primary outcomes of the extension study were safety and mean change in BCVA from month 12 of the Sailing Study to month 12 of the extension study. The results of the study show that conbercept can improve the BCVA in patients with centre-invovled DME, and its efficacy was better than that of traditional ETDRS laser photocoagulation. The safety of conbercept was good with the total occurrence of AEs being similar between the two groups.

In the present study, the sham/conbercept group followed a PRN regimen of conbercept injections after the first injection at baseline and over 12 months, the actual number of injections was 9.5 with a mean improvement of +8.2 letters. The results of the 1-year open-label extension study showed that the efficacy of conbercept was maintained for 24 months, but the number of injections was not significantly reduced. In the extension study after 12 months, patients in the laser/sham group were crossed over and received conbercept PRN. The laser/sham group showed a significant improvement in BCVA after crossing over to conbercept therapy, and there was no difference in BCVA between the two groups at the end of the extension study. This is a different result from other studies where anti-VEGF was delayed and the delayed anti-VEGF group did not catch up to the group treated with anti-VEGF from baseline.

Several studies have demonstrated that anti-VEGF treatment is superior to laser in DME. ${ }^{15-17} 19$ The RISE and RIDE trials compared the efficacy of ranibizumab for DME with PRN laser photocoagulation, and the results showed that ranibizumab improved BCVA, reduced the risk of vision loss and improved the parameters of macular oedema. ${ }^{19}$ The VISTA and VIVID studies examined two regimens of aflibercept versus laser photocoagulation for DME and showed that aflibercept was superior to laser photocoagulation in terms of visual acuity and anatomic changes. ${ }^{15-17}$ Despite using a different anti-VEGF agent, the Sailing Study showed similar results to the RISE, RIDE, VISTA and VIVID trials. Conbercept, which is similar to aflibercept, is a recombinant fusion protein composed of VEGF binding domain from human VEGF receptors 1 and $2 .{ }^{20}$ However, conbercept may have a higher potency and a longer half-life compared with aflibercept because of an additional portion in the fourth binding domain of VEGF receptor 2, which enhances the rate of binding and the stability of the binding complex. ${ }^{21}$ In the 12-month results of aflibercept in the phase III studies, the mean injection numbers of $2 \mathrm{q} 4$ and $2 \mathrm{q} 8$ groups were 11.8 and 8.4 in VISTA, and 12.2 and 8.7 in VIVID, respectively. ${ }^{15}$ In our Sailing Study, the number of conbercept injections was 9.5, which is similar to the number of injections in $2 \mathrm{q} 8$ group. While this number is lower, it is important to recognise that the injection freuqnecy in VIVID and VISTA were fixed not PRN. Of note, at the time of the study, aflibercept and ranibizumab had not been approved for the treatment of DME in China. Thus, additional clinical studies are needed to compare the efficacy of conbercept with other anti-VEGF agents in DME.

The efficacy of laser photocoagulation in improving BCVA during the Sailing Study was similar to that in VISTA and VIVID, with $+0.3 \pm 12.0,+0.2 \pm 12.5$ and $+1.2 \pm 10.6$ letters in BCVA, respectively. In our study, the percentage of eyes that had BCVA improvement in the laser group was greater than similar laser groups in other clinical trials. The reason for this is unknown but may depend on external factors such as the investigators' operational approaches and the type of laser used, as well as the patient population. The factors associated with differences in visual acuity outcomes in eyes treated with panretinal photocoagulation include the HbA1c levels and the severity of the diabetic retinopathy in these patients. ${ }^{22}$ However, our results do indicate that the standard treatment of laser photocoagulation is still a good therapeutic option for some patients, but it is noteworthy 
that the laser group had a higher proportion of eyes with vision loss than the conbercept group. However, the proportion of patients with loss of $\geq 15$ letters in the laser therapy group was $1.1 \%$, far less than the $9.1 \%$ and $10.6 \%$ observed in the VIVID and VISTA trials. ${ }^{15-17}$

During the extension study, patients in the laser group had significant improvement in visual acuity after crossing over to receive PRN conbercept injections and reached similar mean change in BCVA levels compared with patients in the conbercept group at 24 months, suggesting the promising efficacy of conbercept in DME. However, it took 2 months to demonstrate a four-letter BCVA improvement in the laser group, while the conbercept group achieved it in the first month. The delayed benefit from conbercept injection in the laser group could be due to the longer duration of the oedema and resulting outer retinal changes due to the prolonged oedema in the macular tissue. Nonetheless, compared with the RESTORE extension study of ranibizumab where it took nearly 12 months to gain +4 letters after switching to ranibizumab, ${ }^{23}$ conbercept cross-over patients exhibited a faster visual acuity improvement. The rapid and sustained effect of conbercept could improve the quality of life and reduce the risk of vision loss for patients with DME.

With respect to the anatomical outcomes (CRT, TMV and leakage area) and the vision-related quality of life scores (NEI VFQ-25 total score), rapid and sustained improvements were observed in the conbercept group over the first 12 months. The laser group showed worse vision-related quality of life results, despite steady but slow improvement in anatomical indices. In the extension study, CRT in the laser group decreased to a similar level as that in the conbercept group. This supports the fact that conbercept improved the pathological changes in the eye, resulting in better visual acuity and better vision-related quality of life. This was also observed in the RISE, RIDE, VISTA, VIVID and RESTORE trials. ${ }^{15-17} 1923$

In the Sailing Study, the major AEs in the conbercept group were intraocular hypertension and subconjunctival haemorrhage (mostly mild), which were consistent with the AEs reported in previous studies. ${ }^{15-20} 23-25$ Endophthalmitis occurred in two patients after the injection of conbercept, which was aligned with the low incidence of endophthalmitis reported after the injection of saline, ${ }^{26}$ bevacizumab, ${ }^{27}$ ranibizumab ${ }^{19} 28$ and aflibercept. ${ }^{17}$ The incidence of non-infectious intraocular inflammation was $0.12 \%(1 / 861)$ of all injections, within the range of $0.09 \%-0.37 \%$ reported in the literature. ${ }^{29}$ These AEs were predominantly associated with any routine intravitreal injection. The 2-year results of safety were similar to the safety profile of conbercept in the 1-year Sailing Study. No new ocular and nonocular AEs were identified. Overall, the safety of conbercept treatment was similar to other anti-VEGF agents.

A limitation of the extension study is that only $62.6 \%$ $(157 / 251)$ of the initial patients entered the study. Of the 157 subjects enrolled, 142 completed the extension study and 15 withdrew before completing the study. Due to the limited sample size, it is hard to identify the significance of infrequent SAEs that occurred across groups, such as cerebrovascular accident and myocardial infarction.

In conclusion, the $0.5 \mathrm{mg}$ conbercept PRN treatment regimen significantly improved the functional and anatomical outcomes of patients with centre-involved DME, compared with laser photocoagulation. The evidence from the Sailing extension study confirms that conbercept was well tolerated and its efficacy was sustained through 24 months. Intravitreal injections of conbercept can be considered an additional option in the management of patients with DME.

\section{Author affiliations}

Department of Ophthalmology (aka National Clinical Research Center for Eye Diseases), Shanghai General Hospital, Shanghai, China

${ }^{2}$ School of Medicine, Shanghai Jiao Tong University, Shanghai, China

${ }^{3}$ Admin Office, The Eye Specialist Hospital, Shenyang, Liaoning, China

${ }^{4}$ Department of Ophthalmology, Army Medical Center of PLA, Chongqin, China

${ }^{5}$ Department of Ophthalmology, Wuhan General Hospital of Guangzhou Military Region, Wuhan, China

${ }^{6}$ Department of Ophthalmology, Xijing Hospital, Xian, Shaanxi, China

${ }^{7}$ Department of Ophthalmology, Fourth Military Medical University, Xi'an, Shaanxi, China

${ }^{8}$ Department of Ocular Fundus Disease, Wenzhou Medical University Eye Hospital, Wenzhou, Zhejiang, China

${ }^{9}$ Department of Ophthalmology, Wuxi No 2 People's Hospital, Wuxi, Jiangsu, China

${ }^{10}$ Department of Ophthalmology, Third Military Medical University Southwest

Hospital, Chongqing, China

${ }^{11}$ Henan Eye Institute, Henan Eye Hospital, People's Hospital of Zhengzhou University,

Henan Provincial People's Hospital, Zhengzhou, China

${ }^{12}$ Department of Ophthalmology, Peking University Third Hospital, Beijing, China

${ }^{13}$ Department of Ophthalmology, Beijing Tongren Hospital, Beijing, Beijing, China

${ }^{14}$ Department of Ophthalmology, Capital Medical University, Beijing, China

${ }^{15}$ Department of Ocular Fundus Disease, Tianjin Medical University Eye Hospital,

Tianiin, China

${ }^{16}$ Department of Ophthalmology, Shanghai Jiao Tong University School of Medicine Affiliated Renji Hospital, Shanghai, China

${ }^{17}$ Department of Ophthalmology, Sichuan University West China Hospital, Chengdu Sichuan, China

${ }^{18}$ Department of Ophthalmology, The First Affiliated Hospital of Chongqing Medical University, Chongqing, China

${ }^{19}$ Department of Ophthalmology, Peking Union Medical College Hospital, Dongcheng-qu, China

${ }^{20}$ Zhongshan Ophthalmic Center, Sun Yat-Sen University, Guangzhou, Guangdong, China

${ }^{21}$ Department of Ophthalmology, Peking University People's Hospital, Beijing, China

${ }^{22}$ Medical Research Center, Chengdu Kanghong Biotechnology Inc, Chengdu,

Sichuan, China

${ }^{23}$ Medicial Department, Chengdu Kanghong Biotechnology Inc, Chengdu, Sichuan, China

${ }^{24}$ Bascom Palmer Eye Institute, University of Miami Health System, Miami, Florida, USA

${ }^{25}$ Retina, Ophthalmic Consultants of Boston, Boston, Massachusetts, USA

${ }^{26}$ Cole Eye Institute, Cleveland Clinic, Cleveland, Ohio, USA

Contributors Conception and design, obtained funding and overall responsibility: $\mathrm{KL}$ and $X X$; data collection: $\mathrm{KL}$, WH, JiYe, YS, YW, XLiu, ZW, SC, KF, YL, FZ, ZL, LL, JZ, $X Z$, JuYe, XLia and XLi; analysis, interpretation and writing: HW, XK, QW, XW, JL and ST; manuscript revision: PR, JSH and PK.

Funding The study was supported by National Key R\&D Program of China (2016YFC0904800 and 2019YFC0840607) and Chengdu Kanghong Biotechnology Inc. (number 36 ShuxiRoad, JinniuDistrict, Chengdu, China).

Competing interests XK, QW, JL, ST and XW are employees of Chengdu Kanghong Biotechnology, Company, Ltd. PR and PK are consultants to Chengdu Kanghong Biotech.

Patient consent for publication Not required.

Ethics approval The protocols and amendments (including the Sailing Study and its extension study) were approved by the ethics committees of all participating centres and in accordance with Good Clinical Practice and the Declaration of Helsinki. Written informed consent was obtained from each patient. The patients who completed the 12-month Sailing Study signed an additional informed consent form to enter the extension study and had another 12 months of follow-up. The study protocol is available from the authors at drxuxun@sjtu.edu.cn.

Provenance and peer review Not commissioned; externally peer reviewed.

Data availability statement No data are available. This study is under the supervision of the Ministry of Science and Technology of China, and data sharing cannot be provided temporarily.

Supplemental material This content has been supplied by the author(s). It has not been vetted by BMJ Publishing Group Limited (BMJ) and may not have been peer-reviewed. Any opinions or recommendations discussed are solely those of the author(s) and are not endorsed by BMJ. BMJ disclaims all liability and responsibility arising from any reliance placed on the content. Where the content includes any translated material, BMJ does not warrant the accuracy and reliability of the translations (including but not limited to local regulations, clinical guidelines, terminology, drug names and drug dosages), and is not responsible for any error and/or omissions arising from translation and adaptation or otherwise. 
Open access This is an open access article distributed in accordance with the Creative Commons Attribution Non Commercial (CC BY-NC 4.0) license, which permits others to distribute, remix, adapt, build upon this work non-commercially, and license their derivative works on different terms, provided the original work is properly cited, appropriate credit is given, any changes made indicated, and the use is non-commercial. See: http://creativecommons.org/licenses/by-nc/4.0/.

\section{ORCID iD}

Xun Xu http://orcid.org/0000-0002-4246-4343

\section{REFERENCES}

1 American Diabetes Association. 11. Microvascular Complications and Foot Care: Standards of Medical Care in Diabetes-2019. Diabetes Care 2019:42:S124-38.

2 Cheung N, Mitchell P, Wong TY. Diabetic retinopathy. Lancet 2010;376:124-36.

3 Wong TY, Sun J, Kawasaki R, et al. Guidelines on diabetic eye care: the International Council of ophthalmology recommendations for screening, follow-up, referral, and treatment based on resource settings. Ophthalmology 2018;125:1608-22.

4 Yau JWY, Rogers SL, Kawasaki R, et al. Global prevalence and major risk factors of diabetic retinopathy. Diabetes Care 2012;35:556-64.

5 Sivaprasad S, Gupta B, Gulliford MC, et al. Ethnic variations in the prevalence of diabetic retinopathy in people with diabetes attending screening in the United Kingdom (drive UK). PLoS One 2012;7:e32182.

6 Sivaprasad S, Gupta B, Crosby-Nwaobi R, et al. Prevalence of diabetic retinopathy in various ethnic groups: a worldwide perspective. Surv Ophthalmol 2012;57:347-70.

7 Browning DJ, Stewart MW, Lee C. Diabetic macular edema: evidence-based management. Indian J Ophthalmol 2018;66:1736-50.

8 Shah CP, Chen C. Review of therapeutic advances in diabetic retinopathy. Ther Adv Endocrinol Metab 2011;2:39-53.

9 Ferrara N, Gerber H-P, LeCouter J. The biology of VEGF and its receptors. Nat Med 2003;9:669-76.

10 Spilsbury K, Garrett KL, Shen WY, et al. Overexpression of vascular endothelial growth factor (VEGF) in the retinal pigment epithelium leads to the development of choroidal neovascularization. Am J Pathol 2000;157:135-44.

11 van Lookeren Campagne M, LeCouter J, Yaspan BL, et al. Mechanisms of age-related macular degeneration and therapeutic opportunities. J Pathol 2014;232:151-64

12 Homayouni M. Vascular endothelial growth factors and their inhibitors in ocular neovascular disorders. J Ophthalmic Vis Res 2009;4:105-14.

13 Solomon SD, Lindsley K, Vedula SS, et al. Anti-Vascular endothelial growth factor for neovascular age-related macular degeneration. Cochrane Database Syst Rev 2019:3:CD005139

14 Ollendorf DA, Colby JA, Pearson SD. Comparative effectiveness of anti-VEGF agents for diabetic macular edema. Int J Technol Assess Health Care 2013:29:392-401.
15 Korobelnik J-F, Do DV, Schmidt-Erfurth U, et al. Intravitreal aflibercept for diabetic macular edema. Ophthalmology 2014;121:2247-54.

16 Brown DM, Schmidt-Erfurth U, Do DV, et al. Intravitreal aflibercept for diabetic macular edema: 100-Week results from the VISTA and vivid studies. Ophthalmology 2015; 122:2044-52.

17 Heier JS, Korobelnik J-F, Brown DM, et al. Intravitreal aflibercept for diabetic macular edema: 148-Week results from the VISTA and vivid studies. Ophthalmology 2016;123:2376-85.

18 Zhang J, Liang Y, Xie J, et al. Conbercept for patients with age-related macular degeneration: a systematic review. BMC Ophthalmol 2018;18:142.

19 Nguyen QD, Brown DM, Marcus DM, et al. Ranibizumab for diabetic macular edema: results from 2 phase III randomized trials: rise and ride. Ophthalmology 2012;119:789-801.

20 Wang Q, Li T, Wu Z, et al. Novel VEGF decoy receptor fusion protein conbercept targeting multiple VEGF isoforms provide remarkable anti-angiogenesis effect in vivo. PLoS One 2013;8:e70544.

21 Li H, Lei N, Zhang M, et al. Pharmacokinetics of a long-lasting anti-VEGF fusion protein in rabbit. Exp Eye Res 2012;97:154-9.

22 Bressler SB, Beaulieu WT, Glassman AR, et al. Panretinal photocoagulation versus ranibizumab for proliferative diabetic retinopathy: factors associated with vision and edema outcomes. Ophthalmology 2018;125:1776-83.

23 Schmidt-Erfurth U, Lang GE, Holz FG, et al. Three-Year outcomes of individualized ranibizumab treatment in patients with diabetic macular edema: the restore extension study. Ophthalmology 2014;121:1045-53.

24 Liu K, Song Y, Xu G, et al. Conbercept for treatment of neovascular age-related macular degeneration: results of the randomized phase 3 Phoenix study. Am J Ophthalmol 2019;197:156-67.

25 Sun Z, Zhou H, Lin B, et al. Efficacy and safety of intravitreal CONBERCEPT injections in macular edema secondary to retinal vein occlusion. Retina 2017;37:1723-30.

26 Diabetic Retinopathy Clinical Research Network*. Randomized clinical trial evaluating intravitreal ranibizumab or saline for vitreous hemorrhage from proliferative diabetic retinopathy. JAMA Ophthalmol 2013;131:283-93.

27 Ahn J, Woo SJ, Chung H, et al. The effect of adjunctive intravitreal bevacizumab for preventing postvitrectomy hemorrhage in proliferative diabetic retinopathy. Ophthalmology 2011;118:2218-26.

28 Googe J, Brucker AJ, Diabetic Retinopathy Clinical Research Network, et al. Randomized trial evaluating short-term effects of intravitreal ranibizumab or triamcinolone acetonide on macular edema after focal/grid laser for diabetic macular edema in eyes also receiving panretinal photocoagulation. Retina 2011;31:1009-27.

29 Daien V, Nguyen V, Essex RW, et al. Incidence and outcomes of infectious and noninfectious endophthalmitis after intravitreal injections for age-related macular degeneration. Ophthalmology 2018;125:66-74. 